TRANS · núm. I7 2013

DOSSIER $\cdot 35-50$
Los escasos estudios académicos acerca de la industria del doblaje y la falta de unos estándares de calidad en esta actividad han provocado la inexistencia de unas normas profesionales en la traducción para el doblaje en España. Aunque existen ciertas convenciones comunes en todo el país, no hay homogeneidad en aspectos como los símbolos utilizados o las líneas que debe tener un take, sino que cada estudio de doblaje aplica sus propias normas según sus costumbres y necesidades.

El grupo de investigación TRAMA (Traducción y Comunicación en los Medios Audiovisuales) de la Universitat Jaume I de Castellón, ha llevado a cabo un estudio empírico y descriptivo, financiado por el antiguo Ministerio de Educación, con el objetivo de elaborar un mapa de las normas profesionales del doblaje en España. En el presente artículo describiremos dicho estudio y analizaremos los datos obtenidos desde la perspectiva de la profesión del traductor, reflexionando sobre la relevancia que esta cartografía de convenciones reales tiene para la profesión y la formación del traductor-adaptador para el doblaje en España.

PALABRAS CLAVE: doblaje, convenciones, normas matriciales, take, símbolos del doblaje

\title{
Las normas profesionales de la traducción para el doblaje en España
}

\section{Professional Norms for Dubbing in Spain}

To date, few academic studies about the dubbing industry have been conducted and no quality standards have ever been set. As a consequence, translation for dubbing in Spain lacks a set of professional norms. Although Spanish dubbing studios share some common conventions, there is no uniformity in the use of parameters such as dubbing symbols or the number of lines a take must have. Instead, each dubbing studio applies its own rules according to its habits and needs.

The research group TRAMA (Translation and Communication in Audiovisual

Anna Marzà I lbànez

Universitat Jaume I

Gloria TorRalba Miralles

Universitat Jaume I,

Universitat de València

GRUPO TRAMA
Media) from the Universitat Jaume I of Castelló, has conducted an empirical and descriptive study, financed by the former Ministry of Education, with the aim of producing a map of the professional norms for dubbing in Spain.

This article sets out to describe this study and to analyse the data gathered from a professional perspective. A discussion will follow, highlighting the relevance of this cartography of norms for the profession and for the training of translators-adapters in Spain.

KEY WORDS: dubbing, conventions, matricial norms, take, dubbing symbols 


\section{INTRODUCCIÓN}

En este artículo presentamos un estudio financiado por el Ministerio de Ciencia e Innovación y 2010 por el grupo TRAMA ${ }^{\mathrm{I}}$ (Traducción y Comunicación en los Medios Audiovisuales), titulado «Estudio empírico y descriptivo de las normas profesionales de la traducción audiovisual para televisión en España». El objetivo principal del proyecto es la descripción de las normas matriciales (Toury, I995) que rigen la estructura de las traducciones para el doblaje y la subtitulación en España. En el presente artículo nos centraremos en la descripción de las normas profesionales de la traducción para el doblaje.

Una de las características esenciales del doblaje, descrita con detalle por Whitman-Linsen (I992) y Chaume (2003 y 20I2), es el largo proceso que conlleva y el gran número de agentes implicados en su elaboración. En España, aun siendo el país que cuenta con menor número de agentes humanos involucrados en este proceso (Chaume, 20I2), el texto audiovisual pasa por las manos de productor, distribuidor, exhibidor, director del estudio de doblaje (para casting y producción), traductor, adaptador, actores, director y técnicos de doblaje antes de llegar al emisor final, la cadena de televisión o distribuidor.

Por otra parte, la fase específica de traducción para el doblaje conlleva, a su vez, varias tareas diferenciadas: traducción, adaptación y sincronización, segmentación del texto meta e inserción de símbolos. De todas ellas, solo la traducción recae siempre y exclusivamente en el

I TRAMA (http://www.trama.uji.es) es un joven grupo de investigación con sede en la Universitat Jaume I de Castellón, formado por nueve miembros y cuatro colaboradores, dedicado a la docencia, investigación y práctica de la traducción audiovisual en sus diversas modalidades.

traductor. El resto de tareas pueden ser obra del mismo traductor, pero también del asistente de doblaje, del director de doblaje o del adaptador (Ávila, I997; Chaves, 2000; Whitman-Linsen, I992; Gilabert et ál., 200I).

Desde el grupo de investigación TRAMA, como profesionales y formadores de futuros traductores audiovisuales, abogamos por centralizar todas estas tareas en una única figura, la del traductor-adaptador, por motivos económicos y de calidad. Como bien defiende Chaume (2012: 59):

On the one hand, the translator knows the source language and, therefore, knows how far the translation can be manipulated. On the other hand, the process would be faster and cheaper, since the translator would deliver a translation that already meets the demands of dialogue writing, is split into takes, and contains all the dubbing symbols to help talents in their performance.

Nuestro objetivo es formar profesionales completos, capaces de combinar la traducción de textos audiovisuales con el conocimiento profundo del mercado del doblaje y sus normas y un manejo eficaz de la tecnología aplicada al ámbito. Solo este dominio global del proceso de la traducción para el doblaje les permitirá especializarse, ofrecer un producto de altísima calidad y, quizás, llegar a vivir de ello.

No obstante, conocer con detalle y profundidad la práctica del doblaje en España no es tarea fácil:

[..] dubbing is resistant to globalization and still refuses to homogenize its processes. This is maybe due to its consideration as an art form (i.e. unlike subtitling, dubbing professionals would subscribe to the idea that they are taking part in an artistic - rather than linguistic, translational or technologi- 
cal — process, although in my view subtitling is also much more than a mere craft). Local traditions persist, as do differences related to the medium or dissemination, i.e. cinema, television, or DVD (Chaume, ib.: Io).

Históricamente, la traducción y la adaptación para el doblaje han sido profesiones que no se aprendían en el marco de instituciones académicas y, por tanto, no contaban con ningún aparato teórico previo: los traductores audiovisuales aprendían el oficio trabajando, con la experiencia y los consejos de otros traductores, adaptadores y asistentes de doblaje (Chaume, ib.: 65; Gilabert et ál., 200I). La comunicación entre empresas era escasa y ciertas convenciones aleatorias se fueron instaurando a medida que los propios actores y directores trabajaban en los distintos estudios. Incluso hoy en día, con los estudios universitarios de traducción plenamente consolidados y la investigación en traducción audiovisual (TAV) en pleno auge, no existen directrices generalizadas para la práctica profesional del doblaje en España: cada estudio cuenta con sus propias normas de trabajo no escritas e instauradas a lo largo de los años, y el traductor profesional debe pedir que le faciliten las normas imperantes en cada estudio para el que empieza a trabajar. Algunas de estas convenciones son compartidas entre todos los estudios españoles; otras, solo entre los estudios de una misma comunidad autónoma; y otras son totalmente específicas de cada estudio.

Hoy en día, pues, la práctica del doblaje en España conforma un complejo mapa de convenciones compartidas o específicas que nunca ha sido detallado. Los resultados de este estudio van encaminados a definir con mayor precisión estas normas profesionales y, con ello, a proporcionar una herramienta clave para los traductores en activo y para la formación de futuros traductores-adaptadores. A través de la descripción del mencionado mapa de las normas profesionales del doblaje en España, el traductor audiovisual podrá conocer qué parámetros sufren más variación entre estudios y

\section{OBJETIVOS}

Como acabamos de ver, el traductor y el adaptador son solo un eslabón de la larga cadena de agentes implicados en el doblaje. A partir del momento en el que el guión llega a manos de estos profesionales, todos los participantes en el proceso compartirán un mismo código de comunicación y una misma unidad de trabajo, los cuales constituirán la fuente de las normas profesionales que buscamos.

El código de comunicación compartido es el propio guión traducido, elaborado por el traductor y anotado y familiarizado o «domesticado» por el adaptador, en el caso de que sean dos personas diferentes. El guión puede incluir información muy diversa, desde cuestiones paratextuales (títulos en la versión original y doblada; nombre del estudio, traductor, adaptador, etc.) hasta cuestiones propias y específicas de la traducción para el doblaje (anotación de personajes, códigos de tiempo, símbolos, entonación, humor, etc.).

Por otra parte, la unidad de trabajo, aquella que en una sesión de un estudio de doblaje constituye la unidad de grabación, es el take o toma, conocido en otros países dobladores europeos como loop, boucle o anello. Un take es cada uno de los segmentos de texto en los que se divide el guión y, por ello, Whitman-Linsen (I992) los definió como unidades del doblaje y los equiparó a los subtítulos. Esta segmentación del guión en takes responde a normas profesionales, económicas y de narrativa audiovisual, que varían en gran medida según el país pero 
también, hasta cierto punto, dentro de un mismo país.

Así pues, los objetivos específicos del presente estudio son los siguientes:

- La descripción de los criterios de segmentación o corte de takes.

- La descripción del contenido de un take y su formato: numeración, códigos de tiempo, anotación de personajes, etc.

- La descripción de los símbolos utilizados en el doblaje y sus normas de inserción.

- La descripción de los elementos paratextuales que pueden incluirse en el guión para el doblaje y su formato.

- La descripción de la naturaleza y el formato de las anotaciones del traductor-adaptador dirigidas al director o actores de doblaje.

\section{METODOLOGíA}

El ámbito de los estudios profesionales sobre traducción audiovisual no ha sido de los más explorados en el entorno académico. Aun así, cabe destacar las incursiones iniciales en estos aspectos de los cuatro conocidos volúmenes editados en la UPV/EHU de Eguíluz et ál. (I994), Santamaría et ál. (1997), Pajares et ál. (2001) y Merino et ál. (2005). En Kelly (2000), Lorenzo y Pereira (2000 y 200I), Sanderson (2001 y 2002), así como en Duro (200I), se tocan tangencialmente algunos aspectos de esta actividad. Los volúmenes de Díaz Cintas (200I y 2003), Díaz Cintas y Remael (2007), Chaume (2003, 2004 y 20I2) y Del Águila y Rodero (2005) ofrecen un repaso más extenso, por las características de los libros, sobre los aspectos profesionales y sociológicos de la TAV. En el panorama europeo encontramos pinceladas sobre la profesión en los monográficos de Fodor (1976), Ivarsson (1992) y Whitman-Linsen (1992). En la actualidad, no existen grupos nacionales o internacio-

nales que estén llevando a cabo investigación en esta misma materia en TAV.

Así pues, tras el análisis de la citada bibliografía y con la propia experiencia en mente, se pasó al diseño de una encuesta dirigida a los estudios de doblaje. Las cuestiones que queríamos tratar se organizaron en seis bloques:

a) el primer bloque incluye I5 preguntas concretas sobre los criterios de corte de take;

$b$ ) el segundo bloque incluye 4 preguntas sobre I8 cuestiones concretas relativas a la inserción de códigos de tiempo;

c) el tercer bloque presenta 4 preguntas sobre las distintas prácticas de numeración de los takes;

d) el cuarto bloque incluye 9 preguntas sobre las distintas prácticas en la anotación de personajes en las traducciones;

e) el quinto bloque se dedica a averiguar qué símbolos del doblaje se utilizan en las traducciones (se pregunta por el uso de hasta 40 símbolos);

$f$ ) el sexto bloque se centra en las cuestiones paratextuales (títulos en la versión original y doblada, nombre de los agentes del proceso del doblaje, etc.) así como las cuestiones propias y específicas de la traducción para el doblaje (entonación, humor, etc.).

Así pues, la encuesta consta de 39 preguntas, con 2II posibles respuestas o alternativas a las preguntas, lo que ha generado un volumen considerable de contestaciones y una casuística importante en cuanto a las diferentes convenciones usadas para representar las normas matriciales de la traducción para el doblaje. Una vez diseñada, la encuesta se digitalizó con la herramienta de recopilación y análisis de encuestas en línea Survey Monkey. ${ }^{2}$

2 La encuesta final puede encontrarse en: https:// www.surveymonkey.com/s/Z9LLPZ9. 
A continuación se decidió quiénes serían los sujetos encuestados, para lo cual se seleccionaron los estudios de doblaje más representativos de España, siguiendo dos criterios principales: volumen de trabajo e importancia del sector audiovisual en la comunidad autónoma del estudio. Naturalmente, Madrid y Barcelona, como las ciudades más importantes del estado, son las que concentran la mayor parte de la industria del sector y debían estar incluidas. Además, el hecho de que Cataluña cuente con un idioma propio y una televisión autonómica que emite el roo\% de la producción en catalán, hace necesario doblar toda la producción ajena destinada al ente público y, por tanto, cabe esperar que ese segmento de mercado sea importante. Ese es también el caso de la Comunidad Valenciana, Baleares, Euskadi y Galicia, aunque estas comunidades no emiten la totalidad de su programación en catalán, euskera y gallego, respectivamente. Se decidió incluir también los estudios de doblaje de Sevilla, dado que Andalucía cuenta con un sector audiovisual importante que no depende exclusivamente del canal autonómico, sino que también trabaja para un amplio número de productoras y distribuidoras.

En un primer momento se realizó una búsqueda de empresas que podrían estar relacionadas con el sector del doblaje en listados de actividades profesionales en las cámaras de comercio de las distintas comunidades. Sesenta y ocho fueron las empresas que según la descripción podrían dedicarse al doblaje, bien de forma exclusiva o combinada con otras actividades. A todas ellas se les envió una carta de presentación. En esta se preguntaba sobre la posibilidad de concertar una visita al estudio para conocer de cerca sus instalaciones, la manera de trabajar y, por supuesto, pasarles la encuesta en una entrevista personal. Un total de 25 estudios confirmó que se dedicaban al dobla- je y 19 respondieron positivamente a nuestra petición, mientras que los seis restantes explicaron que no podían facilitar la información que necesitábamos. Los miembros del proyecto viajamos a las distintas ciudades donde se encontraban ${ }_{3} 3$ de estos estudios y enviamos el enlace al cuestionario en línea a los 4 restantes que no pudieron recibirnos personalmente.

Es importante destacar que en cada empresa nos atendieron trabajadores diferentes y por tanto no todas las explicaciones provienen del mismo «eslabón» de la cadena de doblaje. Si bien es cierto que en la mayoría de ocasiones las encuestas las rellenaba personal de producción o asistentes de doblaje, contamos con varios cuestionarios respondidos por traductores. $\mathrm{La}$ mayoría de ellos trabajan para estudios valencianos, comunidad en la que las tareas de traducción y ajuste recaen en la misma persona y, por tanto, su experiencia profesional les permite responder a cualquier cuestión relacionada con la fragmentación del guión o la inserción de símbolos. Sin embargo, en otras comunidades autónomas las figuras del traductor y del ajustador son totalmente independientes y el primero puede desconocer por completo las funciones del segundo. Esto nos pasó con uno de los encuestados, el cual, dado que solo cumplía con la labor de traducción, en muchas de las respuestas nos remitía al ajustador o simplemente al estudio de doblaje?

En la visita a los estudios llevamos a cabo las siguientes tareas:

- presentación del proyecto a los jefes de producción de los distintos estudios;

- visita a las instalaciones;

- pase de la encuesta;

- petición de muestras de guiones, traduccio-

3 Por este motivo, en la mayoría de preguntas relacionadas con el ajuste solo contamos con I8 respuestas. 
nes reales, divididas en takes, con símbolos de doblaje y ajustadas, tanto fonética (consonantes labiales y vocales abiertas), como isocrónicamente (equivalencia en la duración de enunciados origen y meta y pausas entre diálogos), que la mayoría de estudios aceptó proporcionarnos.

Una vez finalizada la fase de recopilación de datos, pasamos al vaciado de las encuestas, durante el cual cada miembro del grupo introdujo en la interfaz los resultados obtenidos en los diferentes estudios que había visitado. De esta manera fue posible dejar constancia también de los comentarios que realizaron los diferentes expertos entrevistados así como anotaciones de los encuestadores que servirían para complementar los resultados obtenidos. Así pues, al final del proceso de encuestado, contamos con un gran número de datos obtenidos a través de fuentes y metodologías diversas: entrevistas, cuestionarios, guiones y bibliografía específica. El análisis y contraste de dichos datos funciona como control de calidad de la investigación (Ruiz Olabuénaga, 1996: II3), siguiendo la metodología de triangulación propuesta por la investigación cualitativa (Denzin, 1978).

\section{RESULTADOS}

A continuación presentamos los resultados más destacados de cada uno de los bloques de la encuesta:

\section{Bloque I: Segmentación de takes}

A partir de los resultados del primer bloque, pudimos verificar ciertas convenciones de la traducción para el doblaje citadas por algunos autores como, por ejemplo, el número máximo de líneas por take o por personaje (Chaume, 2004). Así, observamos que el $89,4 \%$ de los encuestados prefería takes de entre ocho y diez líneas. Chaume (ibídem) indica que el número máximo de líneas por take es diez aunque especifica que en Cataluña la cifra se reduce a ocho. Efectivamente, los estudios catalanes marcaron el número ocho en la encuesta, pero no fueron los únicos, ya que también lo hicieron ambos encuestados de Andalucía. Los estudios valencianos fijaron el número máximo en ro y los de Galicia y Madrid en 9 líneas. Los estudios de Euskadi, en cambio, explicaron que admiten takes de hasta II o I2 líneas mientras que los baleares alternan entre 9 y ro líneas.

La pregunta referente al máximo de líneas por personaje arrojó cifras mucho más concretas: $\mathrm{I} 6$ de los $\mathrm{I} 8$ encuestados que respondieron a esta pregunta, marcaron 5 como máximo. Curiosamente, en este caso, los resultados obtenidos no coinciden con la bibliografía publicada sobre convenciones del doblaje: Chaume (ibídem) apunta que también es en Cataluña donde la cifra general de las cinco líneas por personaje se reduce a cuatro.

Evidentemente estos resultados están estrechamente relacionados con el factor económico, ya que los actores de doblaje cobran por dos conceptos diferentes: la llamada "convocatoria» $\mathrm{y}$ el número de takes o fracciones del guión que deben doblarse (Ávila, r997: 54). Así, cuanto mayor sea el número de líneas por take, menos takes tendrá el guión y, por tanto, menos tendrá que pagar el estudio de doblaje a los actores. Solo tres estudios contestaron negativamente

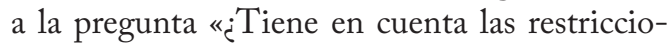
nes económicas a la hora de cortar un take? " y no están afincados en la misma comunidad autónoma, lo que descartaría una posible norma geográfica. Las respuestas afirmativas (un $83,3 \%)$ presentaban un abanico de posibilidades como realizar takes con el máximo número de líneas posibles, no cortar take ante un cambio 
de escena, o intentar que las intervenciones de un mismo personaje se incluyan en una sola toma para evitar que le paguen dos. Esta última fue la respuesta que escogió la mayoría, con un $72,2 \%$. Algún estudio apuntó otros recursos para ahorrar en el número de takes como no marcar los «originales», término usado en la industria para fragmentos extensos sin diálogo, donde la máxima es cortar el take. Efectivamente, el $88,9 \%$ de los encuestados corta toma cuando hay una pausa larga. En cambio, donde no hay homogeneidad es en la duración de dicha pausa. Pedimos que se especificara el número de segundos a partir del cual se corta y las respuestas presentan una horquilla que va desde los io segundos hasta los 30 que indicó un estudio de Baleares.

\section{Bloque 2: Elementos del take}

El segundo bloque se centra en el uso o no de ciertos parámetros y de su inclusión en el guión de doblaje. Los elementos por los que preguntamos son:

- El número de take;

- TCR-in o código de tiempo en el que empieza la primera intervención de un take;

- TCR-out o código de tiempo en el que acaba la última intervención de un take;

- TCR interno o indicativo de cuándo se produce cierta intervención que no es la primera del take;

- Notas;

- Take doble o indicación de que un personaje supera el número máximo de líneas que marca su convenio o su acuerdo con el estudio, que es generalmente cinco;

- v. o. u «original», que como hemos visto en el punto anterior sirve para marcar fragmentos de metraje largos sin diálogo.
A partir de las respuestas obtenidas, se generó el gráfico $\mathrm{I}$.

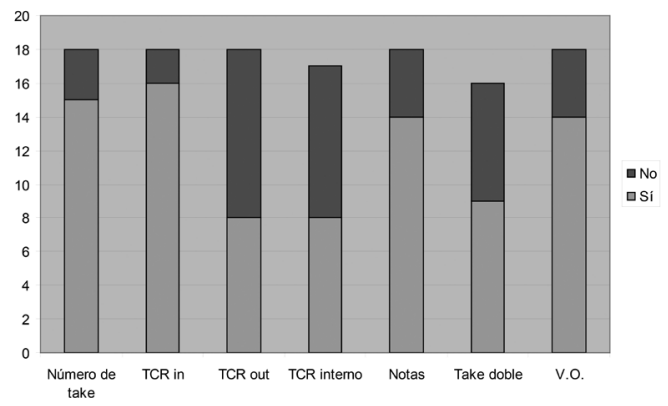

Gráfico I. Parámetros incluidos en los guiones traducidos para el doblaje.

A partir de las respuestas obtenidas, llegamos a la conclusión de que ciertos parámetros son considerados prácticamente imprescindibles. Es el caso del número de take o del TCR de entrada, que casi el 90\% de los encuestados afirma incluir en sus guiones.

Por otra parte, hay mucho menos consenso en el uso del TCR de salida e interno, ya que tanto las respuestas afirmativas como las negativas rondan el 50\% en ambos casos. Contrastando la información de las encuestas con los guiones de doblaje facilitados por los estudios pudimos comprobar cuál es la utilidad mayoritaria de los TCR internos: guiar al actor en intervenciones muy rápidas, diálogos muy seguidos o tras pausas muy largas, pero no señalar el código de tiempo en el que empieza cada declamación.

Lo mismo ocurre con la indicación o no de los takes dobles: de los 16 encuestados que respondieron a esta pregunta, 9 los utilizan, mientras que los estudios de Andalucía, Euskadi y dos de Galicia no lo hacen. La no inclusión de 
este tipo de anotaciones podría deberse a que estas están más relacionadas con la vertiente económica u organizativa que con la artística. Por una parte, un take doble implica que un actor cobrará el doble de la tarifa establecida; y por otra, el número de takes dobles influye en la elaboración del plan de trabajo, ya que las tomas más extensas de lo habitual requieren más tiempo en la sala de doblaje. Esto podría explicar que algunos estudios no vieran necesario hacer visibles estas anotaciones en los guiones y que fuera personal administrativo o programas informáticos específicos los que se encargaran de contar los takes antes de convocar y pagar a los actores.

Por último, un $77,8 \%$ de los encuestados apuntó que se utilizan notas aclaratorias y el mismo porcentaje afirmó indicar en el guión los «originales».

En el siguiente punto del cuestionario preguntamos acerca del formato utilizado en cada uno de los parámetros (negrita, cursiva, mayúscula, entre paréntesis, etc.). Aquí las respuestas son muy variadas y se observa que cada estudio tiene un formato propio.

\section{Bloque 3: Numeración de takes}

Gouadec (2007: 154) asegura que los conocimientos informáticos a veces son tan fundamentales como los lingüísticos o los interculturales. Parece, por tanto, interesante preguntar acerca del uso de recursos informáticos, en concreto de su aplicación a la hora de numerar automáticamente los takes.

Solo I5 de los i9 encuestados contestaron a esta pregunta, ya que, tal y como hemos indicado en el apartado de metodología, a veces el cuestionario lo rellenaba un traductor. Algunos de ellos argumentaron que la numeración de los takes no entraba en sus competencias, sino en las de sus «clientes». Así lo confirman algunos

comentarios acerca de la presencia de programas de numeración automática de takes en los diferentes estudios de doblaje.

A partir las respuestas obtenidas, observamos que el $80 \%$ afirma utilizar recursos informáticos para numerar las tomas. Varios encuestados especifican que suelen recurrir a las macros, mientras que en las entrevistas personales algunos trabajadores de los estudios de doblaje explicaron que han desarrollado sus propios programas tanto para numerar takes como para preparar las plantillas de convocatorias de actores.

\section{Bloque 4: Personajes}

El bloque 4 lo reservamos a preguntas variadas acerca de los nombres de los personajes como, por ejemplo, si se suele recurrir a abreviaturas o motes a la hora de escribirlos en el espacio destinado a ellos, una columna a la izquierda de los diálogos. Sin embargo, queremos destacar otra cuestión, la referente al formato. Ante la pregunta «En la columna de personajes, ¿utiliza un tipo de escritura diferente al de los diálogos?», todos los encuestados afirmaron que escriben el nombre de los personajes en mayúsculas.

Asimismo, es interesante comentar dos respuestas que especifican que delante y detrás del nombre insertan símbolos como el asterisco o el guión. El uso de estos elementos está relacionado con los recursos informáticos para la elaboración de plantillas de doblaje. Así, el programa de la empresa en cuestión identificará como nombres las palabras situadas entre dos símbolos determinados y los computará a la hora de preparar las convocatorias de los actores. Hasta hace poco, de esta tarea se encargaba una persona del departamento de producción, el director de doblaje o incluso los propios traductores; ahora, con la presencia cada vez más 

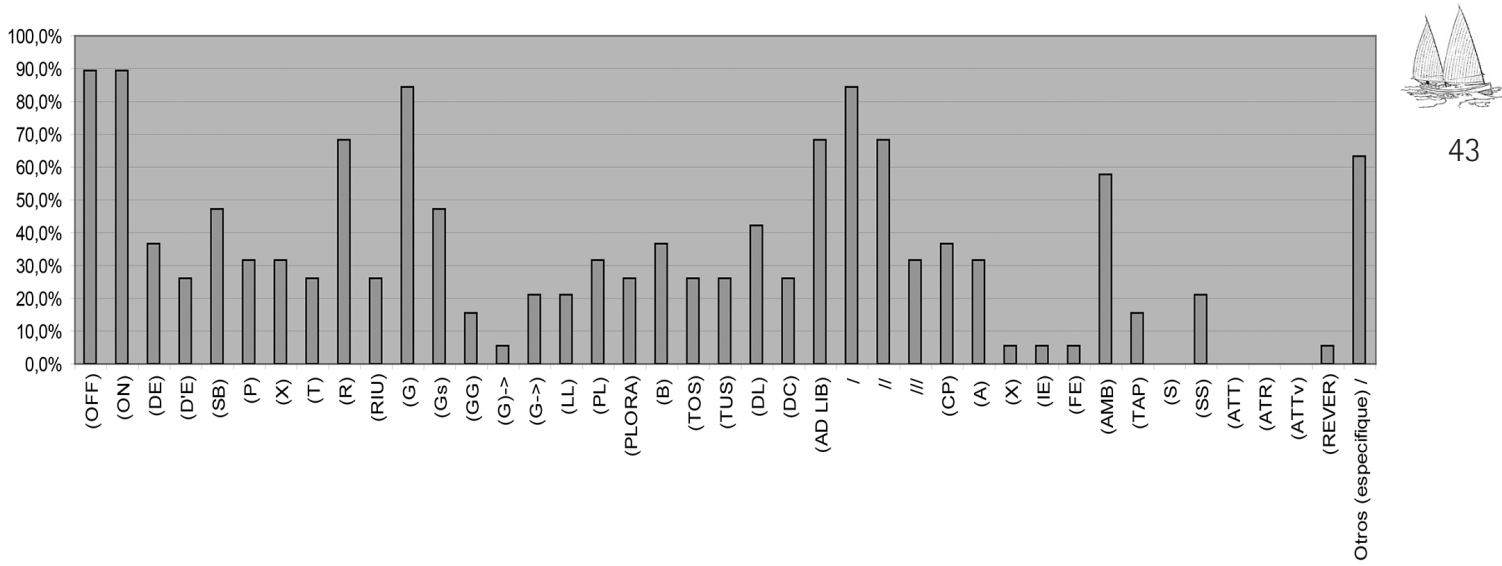

Gráfico 2. Símbolos utilizados en los guiones para el doblaje

habitual de programas desarrollados exclusivamente para cada estudio, como hemos visto en el apartado anterior, las plantillas de doblaje se pueden generar automáticamente a partir del guión traducido y pautado.

\section{Bloque 5: Simbolos}

Chaume (20I2: 80) habla de los símbolos del doblaje en los siguientes términos:

Dialogue writers or dubbing assistants include dubbing symbols in the translation as 'props' to help dubbing actors imitate the screen actors' paralinguistic signs: sounds, pitch, tone, volume, primary voice qualities, etc. They stand for human sound effects (Wright and Lallo 2009: 48-50). The most important symbols refer to where the voice comes from: (ON), (O/s) (On Screen), etc., indicate that the actor is speaking on-screen, i.e., that the audience can see the actor's face or body, whereas (OFF), (v/o), (Off Screen) etc., indicate that the actor is not in field, and the audience cannot see the actor speaking on screen. Other symbols provide more clues to help the dubbing actor.
Dada la importancia de los símbolos en los guiones traducidos para el doblaje, dedicamos a estos signos paralíngüísticos el bloque cinco en su totalidad. Este apartado constaba de una sola pregunta en la que se pedía al entrevistado que marcara todos los símbolos que se utilizaban en el estudio de doblaje para el cual trabajaba. A partir de las respuestas obtenidas, se generó el gráfico 2.

Como puede observarse en el gráfico, los símbolos que obtuvieron los porcentajes más elevados son aquellos que hacen referencia a la presencia o no en pantalla de los actores, hecho que se marca con los símbolos ON y OFF $(89,5 \%)$, a los gestos paralingüísticos (G) y a las pausas (/ o //) (84,2\% cada uno).

Cabe indicar que algunos símbolos se representan de distinta forma según el idioma al que se traduce. Es el caso de (DE) (de espaldas) y (D'E) (d'esquena) o de (P) (pisa), (T) (trepitja) y (x) (xafa), que hace referencia a cuando un personaje empieza a hablar antes de que haya terminado otro, de manera que una intervención se superpone a otra. Así, para este segundo caso, propusimos tres respuestas diferentes, que 
variarían según a qué idioma o variante dialectal se doblara en cada estudio (español, catalán oriental y catalán occidental respectivamente). La suma de los tres porcentajes es de $89,5 \%$, lo cual indica que también es uno de los símbolos más utilizados en los doblajes.

Por otra parte, esta pregunta también sirvió para constatar la utilización de algunos símbolos que, pese a tener un único significado, se representan de distintas maneras. Sucede, por ejemplo, cuando se quiere indicar una serie de gestos: en la encuesta presentamos cuatro símbolos ligeramente diferentes, $(\mathrm{G}) \rightarrow,(\mathrm{G} \rightarrow)$, (Gs) y (GG) y todos ellos fueron seleccionados por algún encuestado, por tanto, se trata de un símbolo con un 100\% de uso, y con cuatro representaciones parecidas, pero diferentes. De ellos, el más común es (Gs), utilizado por un $47,4 \%$.

Como se puede observar en el gráfico, ninguno de los encuestados marcó los símbolos para los técnicos de sonido que proponíamos en la encuesta, ${ }^{4}$ a excepción del de reverberación. No obstante, eso no significa que los ajustadores no incluyan símbolos técnicos. En algunos estudios valencianos y gallegos cuentan con los símbolos (tel/telf), (radio) y (telev) para indicar a través de qué medio habla el personaje. Además, en un estudio de Galicia indican la presencia de música y efectos con el símbolo $(\mathrm{M}+\mathrm{E})$, que tiene una utilidad parecida a (FX), símbolo que aparece en los guiones de un estudio de Baleares para indicar los efectos sonoros.

Las entrevistas personales descubrieron algunas curiosidades como que en Euskadi no solo se utiliza «ad lib» para presentar diálogos de ambiente improvisados sin trascendencia para la historia (su uso más habitual), sino que este símbolo también equivale a cualquier inter-

4 (ATT): el personaje habla a través del teléfono; (ATR): el personaje habla a través de la radio; (ATTV): el personaje habla a través del televisor.

vención paralingüística, lo que generalmente se conoce como "gesto». En estos casos, muchas veces el símbolo va seguido de una especificación, por ejemplo, «tose» o «estornuda». Otros estudios, en cambio, utilizan «ad lib» para matizar la actitud del personaje en cuestión, por ejemplo, «ad lib: en serio».

Por otra parte, el presente estudio ha sacado a la luz una cuestión relevante en cuanto al uso de símbolos, que tal vez requeriría una reflexión más profunda: la gran mayoría de símbolos del doblaje está dirigida a los actores, en un intento de facilitarles la tarea de interpretación e imitación del original. Pensemos que los actores trabajan con tiempos muy ajustados y, por tanto, la claridad e inmediatez de las anotaciones del guión son sumamente importantes. Es de esperar que cada actor prefiera el uso de unos símbolos a otros; algunos agradecerán las indicaciones abundantes mientras que otros tolerarán peor las interrupciones en el guión, $\mathrm{y}$ es muy posible que estas preferencias vengan en gran parte condicionadas por la tradición de cada estudio o de cada comunidad. Dicho de otra forma, la cantidad y variedad de símbolos usados depende en gran medida de su recepción $y$, como veremos a continuación, esta responderá a normas más o menos establecidas por cada estudio.

De media, los estudios trabajan con un listado de 15 símbolos, pero encontramos cifras tan dispares como los 35 símbolos indicados por un estudio de Baleares y los 2 de un estudio de Galicia. Algunos datos relevantes ilustran esta difícil elección entre el uso de abundantes símbolos específicos o pocos y muy generales. En un extremo de personalización, en algunos estudios de Galicia son los propios actores quienes marcan los símbolos de sus intervenciones. En el otro extremo encontraríamos los casos de encuestados (más de un 60\%) que, a pesar de la 
TABla i. Símbolos utilizados POR MÁs del 80\% DE los ESTUdios ENCUESTAdos

\begin{tabular}{ll}
\hline$(\mathrm{ON})$ & el personaje aparece en escena y su boca es perceptible \\
\hline$(\mathrm{OFF})$ & el personaje no aparece en escena \\
\hline$(\mathrm{P}) /(\mathrm{X}) /(\mathrm{T})$ & $\begin{array}{l}\text { el principio de la frase de un personaje «pisa» o se superpone al final de la frase de } \\
\text { otro personaje }\end{array}$ \\
\hline$(\mathrm{R}) /(\mathrm{RIU})$ & risa \\
\hline$(\mathrm{G})$ & $\begin{array}{l}\text { signos paralingüísticos y cualquier gesto humano emitido con la ayuda de las } \\
\text { cuerdas vocales o el aparato de fonación }\end{array}$ \\
\hline$(\mathrm{G}) \rightarrow /(\mathrm{G} \rightarrow) /(\mathrm{Gs}) /(\mathrm{GG})$ & varios gestos seguidos \\
\hline$(/)$ & pausa de unos 4 ó 5 segundos
\end{tabular}

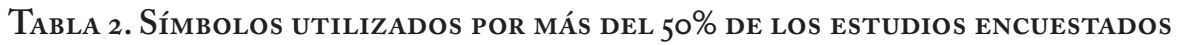

\begin{tabular}{ll}
\hline$(\mathrm{DE}) /(\mathrm{D} ' \mathrm{E})$ & el personaje aparece en pantalla pero está de espaldas \\
\hline$(\mathrm{LL}) /(\mathrm{PL}) /(\mathrm{PLORA})$ & lloro \\
\hline$(/ /)$ & pausa de entre 5 y I5 segundos \\
\hline (AD LIB) & diálogos de ambiente improvisados, sin trascendencia para la historia \\
\hline (AMB) & ambiente \\
\hline
\end{tabular}

gran variedad de símbolos que presentamos en la encuesta, anotaron otros más como (tose) o (grito). También en los comentarios de algunos profesionales se refleja esta oposición: algunos abogan por símbolos variados y específicos que enriquezcan el guión de doblaje y ayuden al actor, mientras que otros defienden una lista reducida.

Si agrupamos los 40 símbolos de nuestro listado por significado, es decir, sin tener en cuenta la variación en las distintas lenguas o las distintas maneras de representar un mismo significado, este número se reduce a 30 , de los cuales 7 son utilizados por más del $80 \%$ de los estudios encuestados. Podríamos considerar, pues, que estos símbolos son universales y su uso es generalizado en la industria del doblaje en España. En la tabla I presentamos dichos símbolos y su correspondiente definición según Chaume (2004: 96-97).

Además, conviene resaltar que hay un segundo grupo de 5 símbolos que utilizan más de la mitad de los estudios (tabla 2).

A juzgar por los resultados, podemos afirmar que existe una lista reducida de símbolos compartida por casi todos los estudios españoles. Prácticamente la mitad de ellos trabaja exclusivamente con los símbolos de dicha lista, mientras que la otra mitad completa el listado con otros más específicos. Cabe destacar, además, que los símbolos que no están presentes en las tablas I y 2 son muy dispares, no se han encontrado demasiadas coincidencias entre estudios, lo que nos lleva a pensar que se trata de anotaciones muy personalizadas que forman parte del lenguaje interno de cada empresa. 


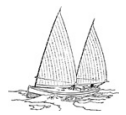

46
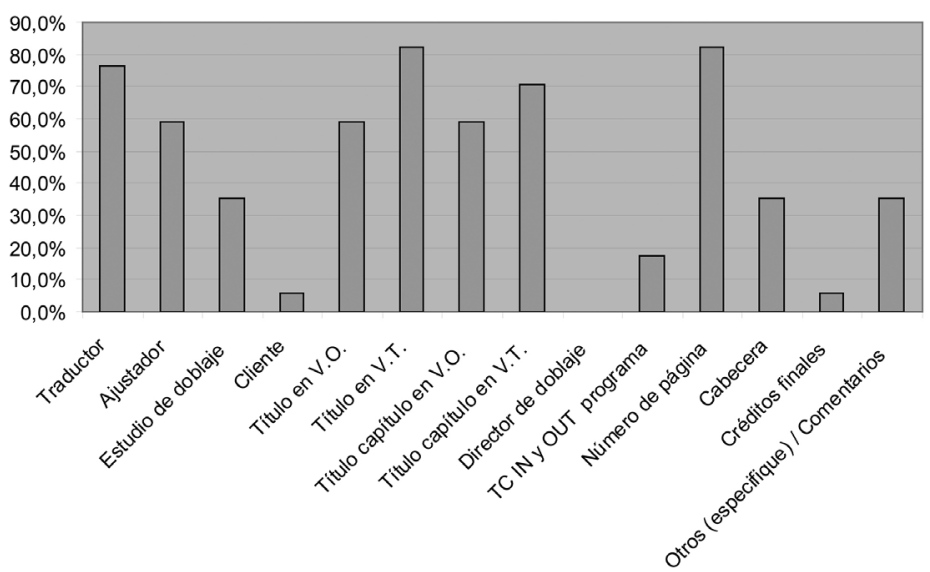

Gráfico 3. Elementos ajenos a los diálogos que se incluyen en los guiones traducidos para el doblaje
Así pues, el uso de los símbolos en la industria del doblaje en España navega entre criterios únicos, compartidos de forma casi universal, y particulares, específicos de cada empresa. No hemos encontrado ninguna recurrencia clara de comportamiento por comunidades autónomas. La única tendencia a la que parecen apuntar los datos es la de un uso más generoso de símbolos en Cataluña y, en cambio, una actitud mucho más comedida en Euskadi, pero esta tendencia debería ser contrastada en futuros estudios.

\section{Bloque 6: Cuestiones paratextuales}

En el último bloque nos centramos en cuestiones paratextuales como, por ejemplo, qué otros datos, aparte de los nombres y los diálogos de los personajes, aparecen en los guiones. Así pues, ofrecimos a los encuestados un listado de estas posibles referencias y les pedimos que marcaran aquellas que se incluyen en los guiones para el doblaje con los que trabajan. En el gráfico 3 presentamos los resultados obtenidos.

Tal y como se desprende del gráfico 3, pudimos observar que no siempre se indica el nombre del programa en la versión original $(58,8 \%$ frente a un $82,4 \%$ de frecuencia de aparición del nombre en la versión traducida); lo mismo ocurre con los códigos de tiempo de inicio y final (I7,6\%), aunque algunos estudios apuntaron que sí que incluyen la duración total del programa.

Nos interesaba particularmente la visibilidad del traductor-ajustador. Algunos autores vienen denunciando el peligro que corre este sector de perder su autoría y otros tipos de consideraciones legales por el hecho de no aparecer en la obra audiovisual (Barambones: 2009: 534) y el poco o nulo reconocimiento que recibe (Chaume, 2004). Por todo ello, nos alegró comprobar que la mayoría de estudios $(76,5 \%)$ trabaja con guiones donde aparece el nombre del traductor. La cifra es sensiblemente más baja en el caso del ajustador $(58,8 \%)$ y esto podría estar relacionado con el hecho de que la tarea de la adaptación en la mayoría de estudios no la realiza una persona ajena al estudio que necesite "visibilizar» en mayor medida su trabajo, sino que se encarga el propio director o asistentes de doblaje, como es el caso de Cataluña. A pesar de todo, el nombre de estos tampoco aparece nunca en los guiones de los estudios encuestados. Por último, cabe indicar que hubo un estudio que no solo seña- 
laba los nombres de estos profesionales, sino también el de los correctores. Una muestra de la sensibilidad por parte del sector que, desgraciadamente, no es la tónica habitual.

\section{CONCLUSIONES}

Los datos presentados en este artículo son solo una muestra de la gran cantidad de información recopilada a través de las encuestas, entrevistas y guiones. No obstante, son suficientes para que surjan interesantes reflexiones con respecto a la práctica profesional del doblaje en España.

Como ya apuntábamos en la introducción, el mapa de las normas profesionales en España constaría de tres niveles: el primer nivel, más global, incluiría aquellas convenciones aceptadas y utilizadas por la gran mayoría de estudios, independientemente de su ubicación geográfica; el segundo nivel abarcaría las convenciones cuyo uso tiene claras delimitaciones geográficas; por último, el tercer nivel lo constituirían las convenciones más particulares, las que tienen una presencia incuestionable en este mapa de normas profesionales pero un uso más fragmentado.

Los resultados muestran que en el panorama de convenciones profesionales de la traducción para el doblaje existe una gran variedad y dispersión de las mismas, como ya esperábamos, pero hemos constatado que no todas las convenciones son aleatorias: en todos los bloques analizados, a excepción del concerniente al corte de takes, hay una mayoría de elementos que responden a criterios particulares y específicos de cada estudio; aun así, en todos encontramos también algún elemento puntual universal. Por ejemplo, en el bloque de elementos del take, solo la inclusión del número de take y el TCR de entrada son universales; o en el bloque de numeración de takes, se constata el uso de la numeración automática en la mayoría de casos, pero su formato no es homogéneo. Precisamente, podemos destacar el formato como el elemento más transversal, presente en todos los bloques analizados, y que concentra la mayor diversidad. El único elemento universal del formato de las traducciones para el doblaje es el uso de mayúsculas para indicar los nombres de los personajes. Por lo demás, cada estudio cuenta con su propio modelo de guión, con formatos totalmente específicos.

E1 bloque que presenta una estructura más clara y homogénea es el relativo a la segmentación de takes, ya que ninguno de sus elementos responde a criterios particulares de los estudios. En otras palabras, se constata un acuerdo respecto al uso de las convenciones de corte de take en España. Se trata, además, del único bloque con elementos que responden a criterios claramente geográficos. Así pues, el número máximo de líneas por toma depende de la comunidad autónoma en la que se encuentre ubicado el estudio. Asimismo, hemos observado que en Euskadi y Andalucía no se indican los takes dobles.

Uno de los resultados más relevantes del estudio, junto con el listado de símbolos más utilizados (véanse tablas I y 2), es la descripción de los elementos que, a pesar de la fragmentación del sector, se han erigido como universales y son utilizados por más del $80 \%$ de los encuestados. Recogemos aquí la lista de estos elementos universales, organizados según los niveles de análisis, con el porcentaje de uso correspondiente:

\section{Segmentación de takes}

-Máximo de 5 líneas por personaje en cada take $(83,3 \%)$

-Aplicación de restricciones económicas en el corte de take $(83,3 \%)$ 
Elementos del take

-Inserción del número de take (83,3\%) y el TCR de entrada de cada take $(88,9 \%)$

48

Numeración de takes

-Uso de recursos informáticos para la numeración de los takes (80\%)

\section{Personajes}

-Uso de la mayúscula para indicar los nombres de los personajes que intervienen en cada take (100\%)

Símbolos

-Uso de los símbolos presentados en la Tabla I $(>80 \%)$

\section{Cuestiones paratextuales}

-No se indican en el guión los nombres de todos los agentes del proceso (traductor, adaptador, director, asistente, actores) (100\%)

-Indicación en el guión del nombre traducido del programa $(82,4 \%)$

- No se indican en el guión los códigos de tiempo de inicio y final $(82,4 \%)$

Otro punto de vista importante que podemos aplicar para el análisis de los datos es el de la formación de traductores, un tema central que se desprende de forma inmediata de los objetivos de este estudio. Los resultados del estudio nos confirman que la formación del traductor audiovisual no estará completa sin el conocimiento del mercado y la cadena del doblaje o sin los conocimientos informáticos adecuados, en consonancia con lo que recoge Cerezo (2012).

El estudio ha demostrado que dos de las fases más importantes en la adaptación del guión, la segmentación del guión en takes y la inserción de símbolos, dependen en gran medida de este conocimiento profundo del mercado y la cadena del doblaje. En este sentido, el hecho de que el documento que crea el traductor-adaptador sea el mismo que utilizará el director, los actores, los técnicos y el estudio de doblaje para la gestión de personal, conlleva una serie de implicaciones añadidas al trabajo del traductor-adaptador. Los datos nos llevan a apuntar la necesidad de que el traductor audiovisual tenga en cuenta las necesidades de los clientes para quien trabaja y de los agentes que le siguen en el proceso del doblaje. Para ello, la formación teórica no es suficiente. Deberíamos cortar con la imagen hessiana que equipara el traductor autónomo al lobo estepario y, desde la formación de traductores, dar la debida importancia a las visitas a los estudios y la presencia en sesiones de grabación. Este contacto directo puede evidenciar las relaciones laborales y económicas que provocan que más de un $80 \%$ de estudios españoles prefiera aplicar las restricciones económicas mencionadas en el análisis durante el proceso de corte de takes. Solo si el traductor-adaptador ha interiorizado estas relaciones y sus implicaciones podrá ofrecer un producto verdaderamente adecuado a su cliente. Asimismo, la inserción de símbolos responde no solo a criterios objetivos deducibles por la información que transmite el texto audiovisual, sino también a la sensibilidad del adaptador respecto a las necesidades de actores, técnicos y director. Unas necesidades que, como hemos visto, variarán en gran medida según el estudio y las personas con las que se trabaje y, por tanto, imposibles de conocer sin un contacto directo y regular con dichos agentes.

Respecto a los conocimientos informáticos, el estudio ha evidenciado que los guiones continúan elaborándose exclusivamente con procesadores de texto, pero hay que destacar que se explotan al máximo los recursos que estas herramientas proporcionan, como las plantillas 
o las macros. También hemos constatado el uso cada vez mayor de programas desarrollados específicamente para los estudios que asumen ciertas tareas como la numeración de takes o la preparación de plantillas para convocar a los actores.

Nos reafirmamos, pues, en la necesidad de formar profesionales completos, con dominio integral de las fases de traducción y adaptación, conocimientos informáticos adecuados al ámbito y que desarrollen la sensibilidad necesaria hacia los diferentes agentes del proceso del doblaje. De esta forma aumentamos sus opciones de encontrar empleo, ya que pueden trabajar como traductores exclusivamente, ofrecer además sus servicios como adaptadores o, incluso, ser contratados por algún estudio para realizar tareas de asistente de doblaje, como el corte de takes, la inserción de ciertos símbolos, la gestión de las sesiones de doblaje o de los programas específicos.

RECIBIDO EN NOVIEMBRE DE 2012 ACEPTADO EN ENERO DE 2013 VERSIÓN FINAL DE NOVIEMBRE DE 2012

\section{REFERENCIAS BIBLIOGRÁFICAS}

Ávila, A. (1997). El doblaje. Madrid: Cátedra.

Barambones, J. (2009). La traducción audiovisual en ETB-I: Estudio descriptivo de la programación infantil y juvenil, tesis doctoral presentada en la Facultad de Letras de la Universidad del País Vasco-Euskal Herriko Unibertsitatea.

Cerezo, B. (2012). La didáctica de la traducción audiovisual en España: un estudio de caso empiricodescriptivo, tesis doctoral presentada en la Facultat de Ciències Humanes i Socials de la Universitat Jaume I.

Chaume, F. (2003). Doblatge i subtitulació per a la TV. Vic: Eumo.

Chaume, F. (2004). Cine y traducción. Madrid: Cáte- dra.

Chaume, F. (2012). Audiovisual Translation: Dubbing. Manchester: St. Jerome.

Chaves, M.J. (2000). La traducción cinematográfica. El doblaje. Huelva: Publicaciones de la Universidad de Huelva.

Del Águila, M.E. y Rodero, E. (2005). El proceso de doblaje take a take. Salamanca: Publicaciones Universidad Pontificia.

Denzin, N. (1978). The research act. A theoretical introduction to sociological methods. Nueva York: Editorial McGraw-Hill.

Díaz Cintas, J. (200I). La traducción audiovisual. El subtitulado. Salamanca: Almar.

Díaz Cintas, J. (2003). Teoría y práctica de la subtitulación. Inglés-español. Barcelona: Ariel.

Díaz Cintas, J. y Remael, A. (2007). Audiovisual Translation: Subtitling. Manchester: St Jerome.

Duro, M. (coord.) (200I). La traducción para el doblaje y la subtitulación. Madrid: Cátedra.

Eguíluz, F. et ál. (eds.) (I994). Trasvases Culturales: literatura, cine, traducción. Gasteiz: Universidad del País Vasco-Euskal Herriko Unibertsitatea.

Fodor, I. (1976). Film Dubbing: Phonetic, Semiotic, Esthetic and Psychological Aspects. Hamburgo: Helmut Buske.

Gilabert, A., Ledesma, I. y Trifol, A. (200I). «La sincronización y la adaptación de guiones cinematográficos». En Duro, M. (coord.). La traducción para el doblaje y la subtitulación. Madrid: Cátedra, pp. 325-330.

Gouadec, D. (2007). Translation as a Profession. Ámsterdam: John Benjamins.

Ivarsson, J. (1992). Subtitling for the Media. A Handbook of an Art. Estocolmo: Transedit.

Kelly, D. (ed.) (200o). La traducción y la interpretación en España hoy: perspectivas profesionales. Granada: Comares.

Lorenzo, L y Pereira, A.M. (eds.) (200o): Traducción subordinada (I). El doblaje. Vigo: Publicacións da Universidade de Vigo.

Lorenzo, L y Pereira, A.M. (eds.) (20or): Traducción subordinada (II). El subtitulado. Vigo: Publicacións da Universidade de Vigo.

Merino, R., Santamaria, J.M. y Pajares, E. (eds.) (2005). Trasvases Culturales: literatura, cine, traducción 4. Gasteiz: Universidad del País VascoEuskal Herriko Unibertsitatea.

Pajares, E., Merino, R. y Santamaria, J.M. (eds.) 
(200I). Trasvases Culturales: literatura, cine, traducción 3. Gasteiz: Universidad del País VascoEuskal Herriko Unibertsitatea.

Ruiz Olabuénaga, J.I. (1996). Metodología de la investigación cualitativa. Bilbao: Universidad de Deusto.

Sanderson, J. (ed.) (200I). ¡Doble o nada! Actas de las I y II Jornadas de doblaje y subtitulación. Alicante: Publicacions de la Universitat d'Alacant.

Sanderson, J. (ed.) (2002). Traductores para todo. Actas de las III Jornadas de doblaje y subtitulación. Alicante: Publicacions de la Universitat d'Alacant.

Santamaría, J.M., Eguíluz, F., Olsen, V., Merino, R., Pajares, E. (eds.) (1997). Trasvases Culturales: literatura, cine, traducción 2. Gasteiz: Universidad del País Vasco-Euskal Herriko Unibertsitatea.

Toury, G. (1995). Descriptive Translation Studies and Beyond. Amsterdam/Filadelfia: John Benjamins.

Whitman-Linsen, C. (1992). Through the Dubbing Glass. Frankfurt: Peter Lang. 